Article

\title{
Liquid-Assisted Grinding to Prepare a Cocrystal of Adefovir Dipivoxil Thermodynamically Less Stable than Its Neat Phase
}

\author{
Sungyup Jung, Insil Choi and Il Won Kim* \\ Department of Chemical Engineering, Soongsil University, Seoul 06978, Korea; \\ E-Mails: yubjjung@soongsil.ac.kr (S.J.); cisq@nate.com (I.C.) \\ * Author to whom correspondence should be addressed; E-Mail: iwkim@ssu.ac.kr; \\ Tel.: +82-2-820-0614; Fax: +82-2-812-5378.
}

Academic Editor: Sitaram Velaga

Received: 13 September 2015 / Accepted: 12 November 2015 / Published: 19 November 2015

\begin{abstract}
Liquid-assisted grinding was employed to generate a cocrystal of adefovir dipivoxil (AD) and glutaric acid (GLU), which had not been successfully obtained through solution crystallization. The cocrystal formation was confirmed with powder X-ray diffraction, and its thermal stability and release behavior were studied through differential scanning calorimetry and dissolution experiments, respectively. The AD/GLU cocrystal was less stable than neat $\mathrm{AD}$ phase and the previously reported $\mathrm{AD}$ cocrystals with other dicarboxylic acids, such as suberic acid and succinic acid. This suggests that the intermolecular interactions of the $\mathrm{AD} / \mathrm{GLU}$ cocrystal are probably weaker than the other crystal phases. The release behavior of the AD/GLU was comparable with the cocrystal with suberic acid. The current study verifies the effectiveness of the liquid-assisted grinding for the preparation of the thermodynamically less stable cocrystal phase.
\end{abstract}

Keywords: cocrystal; grinding; adefovir dipivoxil; stability; release behavior

\section{Introduction}

Crystallization of active pharmaceutical ingredients (APIs) is the process that determines the physical properties of the API solid phases. It is especially important for the orally delivered drugs, where the API crystals are directly used in pharmaceutical dosage forms, such as tablets and capsules. The properties affected by the crystal structures include solubility, stability, and mechanical properties [1-3]. 
Cocrystallization is a relatively recent tool to control the properties of the API crystals, compared to, for example, salt formation and polymorph selection [4,5]. In general, the basic building block of a pharmaceutical cocrystal is the strongly interacting molecules of an API and a cocrystal former (coformer), although cocrystals of two different APIs and multicomponent systems have been also studied [6-8]. A large degree of freedom to select the coformers and the subsequent diversity of the cocrystals have made the cocrystallization an attractive strategy to design the structures and properties of API crystals, even when the coformers are limited as those approved by the regulatory authorities [9].

Some of the processes to prepare pharmaceutical cocrystals are solution crystallization, grinding, sublimation, and so on [5,10]. Among these, the traditional solution crystallization appears the industrially preferable method because the process is well established, easily scalable, and less prone to contamination $[11,12]$. However, there were cases where the solution method was not successful in yielding cocrystals, such as caffeine/glutaric acid, paracetamol/oxalic acid, and itraconazole/malonic acid, in their pure forms, which could be obtained through liquid-assisted grinding [13-15].

We have studied the cocrystallization of adefovir dipivoxil (AD, Figure 1), a prodrug of a broad-spectrum antiviral known as adefovir, mainly through the solution process [16]. Cocrystals of $\mathrm{AD}$ and suberic acid (SUB) formed from both methanol and ethanol solutions, and those of AD and succinic acid formed from ethanol solutions. However, AD cocrystals with glutaric acid (GLU) could not be obtained in their pure form through the solution process. In the present study, liquid-assisted grinding was utilized to prepare the AD/GLU cocrystals, and their thermal stability and release behavior were compared with those of AD/SUB cocrystals as well as neat AD crystals.

(a)

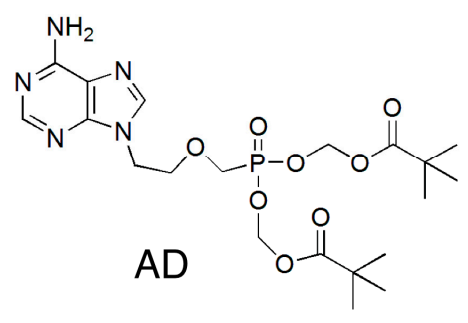

(b)

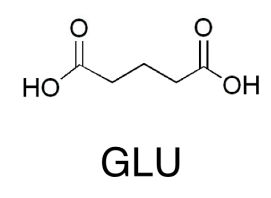

(c)

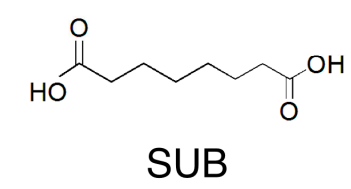

Figure 1. Chemical structures: (a) adefovir dipivoxil (AD); (b) glutaric acid (GLU); (c) suberic acid (SUB).

\section{Results and Discussion}

\subsection{Cocrystal Formation}

AD cocrystals with GLU and SUB were produced by liquid-assisted grinding, and their morphologies were summarized in Figure 2. As a comparison, the neat AD crystals before grinding were also shown in Figure 2a,b. Morphologies of GLU and SUB raw materials were displayed in Figure S1 (Supplementary Materials). OM and SEM micrographs were shown to exhibit overall configurations and individual shapes of crystals, respectively. AD/GLU cocrystals were largely aggregated (Figure 2c) in ca. 100-200 $\mu$ m clusters. Closer observation with SEM revealed that the individual crystals were of a platelet shape (Figure 2d). Their overall size was diverse with the long axes ca. 2-20 $\mu \mathrm{m}$, but their thickness was relatively uniform at about $1-2 \mu \mathrm{m}$. While the overall shapes of AD raw material (Figure 2a,b) somewhat resembled the $\mathrm{AD} / \mathrm{GLU}$ crystals, its individual crystals appeared thinner 
$(0.5-1 \mu \mathrm{m})$ and more loosely attached to each other with visible gaps between large surfaces. In contrast, $\mathrm{AD} / \mathrm{SUB}$ appeared better dispersed with many crystals of sub-micron size, although some bigger crystals were also noticeable (Figure 2e,f). (Note that the shapes of SUB and GLU crystals (Figure S1, Supplementary Materials) did not show clear correlations with their respective cocrystals.) In general, the breakage of particles during milling is related to the maximum fracture energy, Young's modulus, Poisson ratio, hardness as well as initial particle size $[17,18]$. Fundamentally, these materials properties originate from the intermolecular interactions that govern the crystal structures and determine responses when subject to mechanical stress $[14,19]$. In the present study, further analysis on the different morphologies of AD cocrystals formed by grinding was hindered by the lack of AD/GLU crystal structure. Also, more defined milling process needs to be employed to further investigate the correlation between the structure and morphology of the cocrystals.
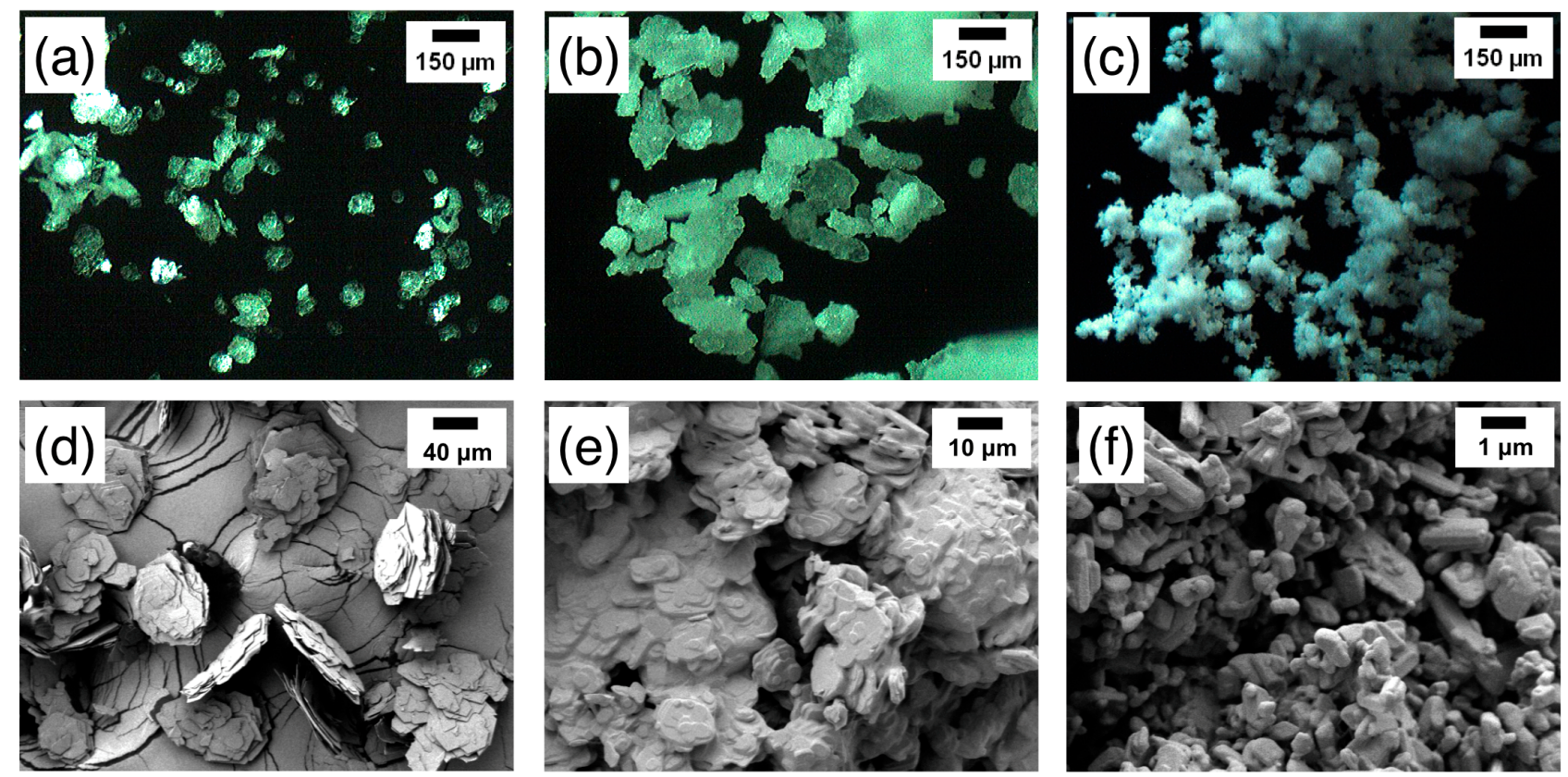

Figure 2. Optical micrographs of $\mathrm{AD}$ (a), $\mathrm{AD} / \mathrm{GLU}$ (b), and $\mathrm{AD} / \mathrm{SUB}$ (c); scanning electron micrographs of $\mathrm{AD}$ (d), $\mathrm{AD} / \mathrm{GLU}$ (e), and $\mathrm{AD} / \mathrm{SUB}$ (f).

The XRD pattern of the AD/GLU cocrystal was compared with those of neat AD and GLU in Figure 3. The diffraction pattern of $\mathrm{AD} / \mathrm{GLU}$ was distinctively different from those of $\mathrm{AD}$ and GLU. Some of the most characteristic diffraction peaks were at $10.54^{\circ}, 17.16^{\circ}, 17.76^{\circ}, 20.70^{\circ}$ and $25.94^{\circ}$ (marked by asterisks). We note here that the diffraction pattern of $\mathrm{AD} / \mathrm{GLU}$ is also different from $\mathrm{AD}$ methanol solvate. For example, the AD/GLU peaks at $10.54^{\circ}, 20.70^{\circ}$ and $25.94^{\circ}$ and the methanol solvate peaks at $16.5^{\circ}, 19.4^{\circ}$ and $25.4^{\circ}$ were mutually exclusive [20]. Overall, the XRD analysis confirmed that a new crystal phase was obtained through the liquid-assisted grinding. Since the diffraction pattern does not match any of the known polymorphs of AD and GLU, the obtained phase is mostly likely the $\mathrm{AD} / \mathrm{GLU}$ cocrystal, which could not be successfully acquired from solution crystallization. This was also in part supported by the sharp melting point of the crystal as shown in the next section, which suggested the formation of a pure phase. 

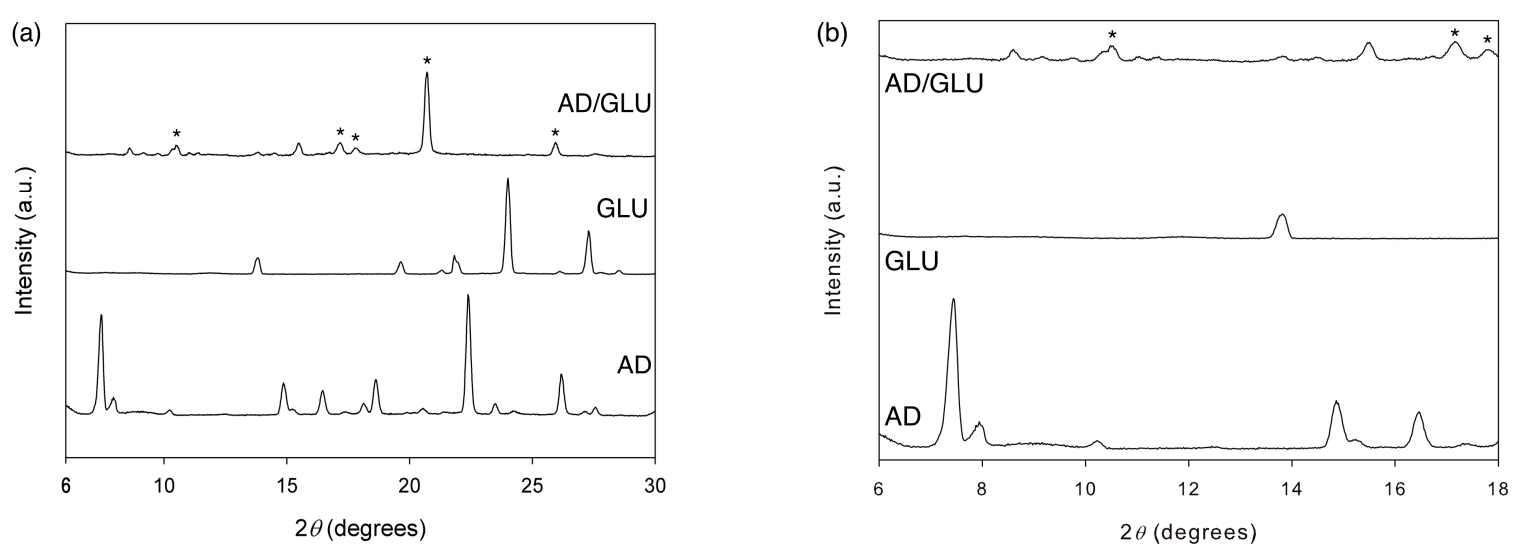

Figure 3. X-ray diffraction (XRD) patterns of the AD/GLU cocrystal and its raw materials (AD and GLU): $2 \theta$ regions of $6^{\circ}-30^{\circ}$ (a) and $6^{\circ}-18^{\circ}(\mathbf{b})$. Some characteristic peaks of the $\mathrm{AD} / \mathrm{GLU}$ cocrystal were marked by asterisks.

Figure 4 was shown to verify the validity of the liquid-assisted grinding in the formation of $A D$ cocrystals. The XRD patterns of the AD/SUB crystals grown through the solution crystallization and liquid-assisted grinding matched nearly perfectly in their peak positions as previously reported [21]. Also shown is the calculated pattern from the single crystal X-ray data [16].

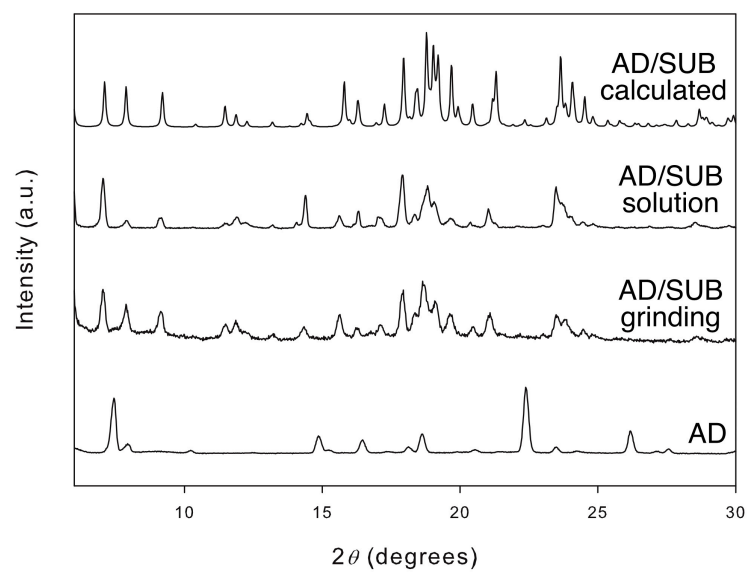

Figure 4. $\mathrm{XRD}$ patterns of neat $\mathrm{AD}$ and $\mathrm{AD} / \mathrm{SUB}$ cocrystals. The pattern of the cocrystal calculated from the known crystal structure was compared with those of the crystals prepared by the grinding method and solution growth as previously reported [21].

\subsection{Thermal Stability and Release Behavior}

Thermal stability of the prepared crystals could be studied through their melting, since there was no premature thermal degradation of the constituent molecules or loss of small molecules (such as water and solvents). Melting behavior of AD/GLU observed with DSC (Figure 5) was compared with those of $\mathrm{AD}$ and $\mathrm{AD} / \mathrm{SUB}$, which had been previously reported [16,21]. The melting points were in the order: $\mathrm{AD} / \mathrm{GLU}\left(88.9^{\circ} \mathrm{C}\right)<\mathrm{AD}\left(93.3^{\circ} \mathrm{C}\right)<\mathrm{AD} / \mathrm{SUB}\left(133.9^{\circ} \mathrm{C}\right)$, and the melting enthalpy was also in the same order: AD/GLU $(60.8 \mathrm{~J} / \mathrm{g})<\mathrm{AD}(75.4 \mathrm{~J} / \mathrm{g})<\mathrm{AD} / \mathrm{SUB}(107.4 \mathrm{~J} / \mathrm{g})$. The melting point of $\mathrm{AD} / \mathrm{GLU}$ cocrystal was lower than those of its pure individual components ( $\mathrm{T}_{\mathrm{m}}$ and enthalpy of GLU: $99.8{ }^{\circ} \mathrm{C}$ and $161.0 \mathrm{~J} / \mathrm{g}$, see Figure S2 in Supplementary Materials), whereas AD/SUB had a melting 
point between those of its components ( $\mathrm{T}_{\mathrm{m}}$ and enthalpy of SUB: $143.8^{\circ} \mathrm{C}$ and $162.6 \mathrm{~J} / \mathrm{g}$, see Figure $\mathrm{S} 2$ in Supplementary Materials). In fact, other previously reported AD cocrystals with saccharin or succinic acid had their melting points between their respective cocrystal components [22,23]. The unique melting behavior of $\mathrm{AD} / \mathrm{GLU}$ suggested that its crystal structure could possess a dramatically different structure (weaker intermolecular interactions) from other AD cocrystals, although the unsolved crystal structure of $\mathrm{AD} / \mathrm{GLU}$ made the conclusion speculative at this moment. Similar phenomenon was seen in other API cocrystals using 4,4'-bipyridine as a coformer [24]. The cocrystal with asprin possessed a crystal structure of a channel inclusion, and it had a lower melting point than its individual components. Other cocrystals with ibuprofen and flurbiprofen had herringbone packing structures, and their melting points were higher than those of their respective cocrystal components.

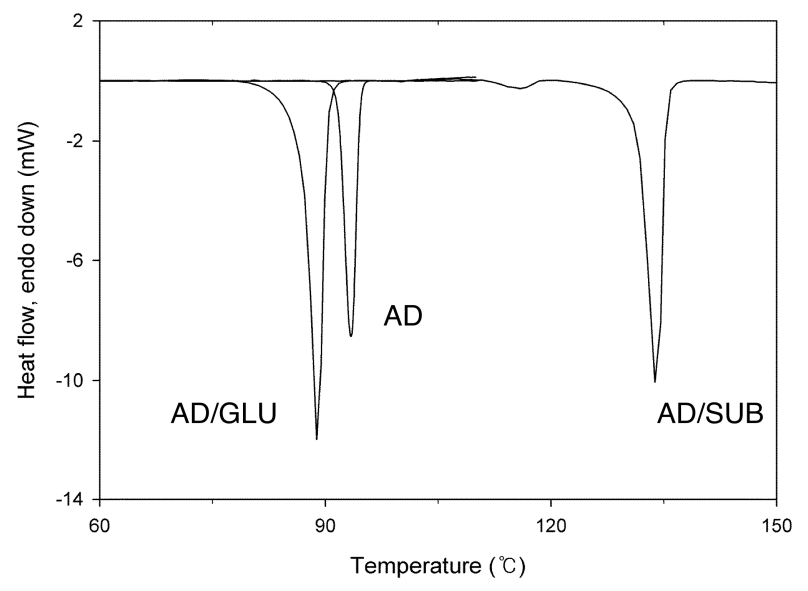

Figure 5. Differential Scanning Calorimetry (DSC) thermograms of AD and its cocrystals. The melting point of AD/GLU was compared with those of $\mathrm{AD}$ and $\mathrm{AD} / \mathrm{SUB}[16,21]$.

We note here that a previously unnoticed small melting peak was found for the AD/SUB cocrystal at $115.9^{\circ} \mathrm{C}(6.6 \mathrm{~J} / \mathrm{g})$, which was still at significantly higher temperature than the melting point of AD. This was possibly related to the crystals with structural defects caused during the milling process, because a similar endothermic peak was not observed with the crystals prepared through solution crystallization [16]. The portion of the crystals was estimated $c a .6 \%$ when only enthalpy values were roughly considered. No such melting behavior related to the probable structural defects was observed for AD/GLU cocrystals. Further detailed studies with controlled processing parameters would be necessary to confirm the correlation between the stability and structural defects.

Release behaviors of $\mathrm{AD}$ cocrystals and $\mathrm{AD}$ raw material were compared in Figure 6. In general, $\mathrm{AD}$ cocrystals showed better release behaviors than $\mathrm{AD}$ raw material. This is consistent with the previous solubility study, and it is probably because hydrogen bonding is the major interaction between $\mathrm{AD}$ and coformers that contribute to the structural integrity of the cocrystals [16]. Also, $\mathrm{AD} / \mathrm{SUB}$ cocrystal showed initially very fast release rate, which could be related to the combination of smaller crystal size (Figure 2) and the portion of defected crystal structure as discussed with melting (Figure 5). Dissolution rate of particles is generally regarded as a function of surface area, diffusion constant, boundary layer thickness as well as solubility $[25,26]$. The current method of liquid-assisted grinding was not controlled in terms of the particle size and shape that could affect the surface area and boundary layer thickness, and the particle-aggregated structures could also modify the diffusion of 
AD molecules by affecting the hydrodynamic properties. Therefore, the current release results should be considered only preliminary. Nonetheless, they suggest that the cocrystal formation based on grinding method is a potentially useful method to regulate API release behavior.

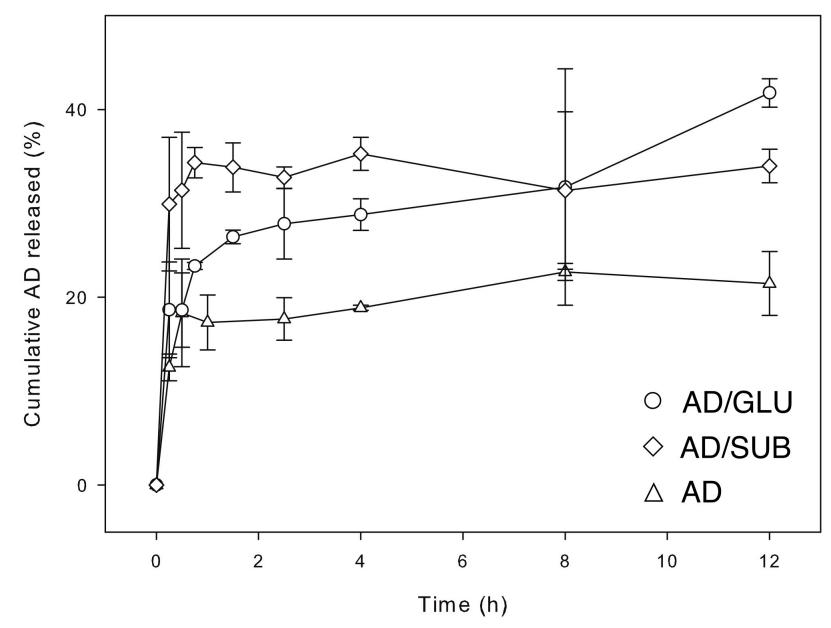

Figure 6. Release behaviors of AD and its cocrystals.

\section{Experimental Section}

AD (L-form, >99\%) was obtained from Amore Pacific Co. (Yongin, Korea). GLU (99\%) and SUB (98\%) were purchased from Sigma-Aldrich (Milwaukee, WI, USA). Methanol (HPLC grade) was from J.T.Baker (Center Valley, PA, USA).

Cocrystallization of $\mathrm{AD}$ and coformers (GLU or SUB) was performed through liquid-assisted grinding. $\mathrm{AD}$ and coformer was mixed in 1:1 mole ratio, and the mixture was ground using an agate mortar and pestle for 20-30 min. AD/GLU and AD/SUB cocrystals were prepared in 0.20 and $0.40 \mathrm{mmol}$ scale, respectively. Methanol was added dropwise over the course of grinding $(0.5 \mathrm{~mL} / \mathrm{mmol}$ cocrystal). After grinding, the cocrystals were dried in a vacuum oven (J-DV01; JISICO, Seoul, Korea) at $40{ }^{\circ} \mathrm{C}$ for $24 \mathrm{~h}$ before further characterization.

Morphologies of $\mathrm{AD}$ and its cocrystals were microscopically observed. Gross morphologies were surveyed with OM using a BX51 microscope (Olympus, Tokyo, Japan) in the reflectance mode with cross polarization. Detailed morphologies were observed with FE-SEM using an Auriga microscope (Carl Zeiss, Oberkochen, Germany) after thin platinum coating to minimize charging.

XRD was performed to identify the crystal phases. A D8 Discover diffractometer (Bruker AXS, Billerica, MA, USA) was used in the $2 \theta-\theta$ mode to scan the $2 \theta$ region of $6^{\circ}-40^{\circ}$ with a scanning rate of $1 \% \mathrm{~min}$. CuKa radiation $(\lambda=1.5406 \AA)$ at $40 \mathrm{kV}$ and $40 \mathrm{~mA}$ was employed.

DSC was utilized to assess the melting properties of the AD and its cocrystals. A DSC821e (Mettler-Toledo, Columbus, $\mathrm{OH}$, USA) was employed under $\mathrm{N}_{2}$ with a scanning rate of $10{ }^{\circ} \mathrm{C} / \mathrm{min}$. The instrument was pre-calibrated using indium for enthalpy and temperature.

The in vitro release behaviors of the $\mathrm{AD}$ and its cocrystals were measured using a dissolution tester (USP type 2 paddle apparatus). A KDT-600 (Kukje Engineering Co., Seoul, Korea) was used under a sink condition. A typical experiment consisted of $750 \mathrm{mg}$ crystal powders in a $300 \mathrm{~mL}$ aqueous solution $\left(\mathrm{pH} 6,37^{\circ} \mathrm{C}\right)$ stirred at $100 \mathrm{rpm}$. Sampling $(5 \mathrm{~mL})$ was done until $12 \mathrm{~h}$ at pre-determined time 
points, and a fresh $5 \mathrm{~mL}$ solution was added to the system after each sampling. Each sampled solution was filtered through a syringe filter of $0.45 \mu \mathrm{m}$ pore size (MFS-13; Avantec, Dublin, CA, USA), and its UV absorbance was measured at $260 \mathrm{~nm}$ using a JASCO (Tokyo, Japan) V-550 UV-Vis spectrophotometer. AD concentration was calculated using a pre-constructed calibration curve.

\section{Conclusions}

In summary, liquid-assisted grinding was utilized to obtain the AD/GLU cocrystal that was difficult to isolate in its pure form through solution crystallization. It had lower thermal stability than neat AD as well as other $\mathrm{AD}$ cocrystals, which had been successfully prepared through solution crystallization. The low enthalpy of fusion of $\mathrm{AD} / \mathrm{GLU}$ suggests that the intermolecular bonding that holds its structure could be much weaker than those in neat AD and the other AD cocrystals, although further investigation would be needed to clarify the exact nature of the intermolecular interactions. The release behavior of the $\mathrm{AD} / \mathrm{GLU}$ was comparable with $\mathrm{AD} / \mathrm{SUB}$, and some improvement appears possible if its particle processing is optimized. The current study demonstrates the usefulness of the grinding method to prepare a thermodynamically less stable AD cocrystal phase, and similar findings are expected in the future for other APIs.

\section{Acknowledgments}

This research was supported by Basic Science Research Program through the National Research Foundation of Korea (NRF) funded by the Ministry of Education (NRF-2013R1A1A2012036).

\section{Author Contributions}

Sungyup Jung performed the bulk of the experimental work. Insil Choi performed some particle characterization. Il Won Kim designed and supervised the work. Sungyup Jung and Il Won Kim analyzed the data, and wrote the manuscript.

\section{Conflicts of Interest}

The authors declare no conflict of interest.

\section{References}

1. Rodríguez-Spong, B.; Price, C.P.; Jayasankar, A.; Matzger, A.J.; Rodríguez-Hornedo, N. General principles of pharmaceutical solid polymorphism: A supramolecular perspective. Adv. Drug Deliv. Rev. 2004, 56, 241-274.

2. Llinàs, A.; Goodman, J.M. Polymorph control: Past, present and future. Drug Discov. Today 2008, 13, 198-210.

3. Saal, C. Selection of solid-state forms: Challenges, opportunities, lessons learned and adventures from recent years. J. Pharm. Pharmacol. 2015, 67, 755-756.

4. Jones, W.; Motherwell, W.D.S.; Trask, A.V. Pharmaceutical cocrystals: An emerging approach to physical property enhancement. MRS Bull. 2006, 31, 875-879. 
5. Shan, N.; Zaworotko, M.J. The role of cocrystals in pharmaceutical science. Drug Discov. Today 2008, 13, 440-446.

6. Lu, J.; Rohani, S. Synthesis and preliminary characterization of sulfamethazine-theophylline co-crystal. J. Pharm. Sci. 2010, 99, 4042-4047.

7. Grobelny, P.; Mukherjee, A.; Desiraju, G.R. Drug-drug co-crystals: Temperature-dependent proton mobility in the molecular complex of isoniazid with 4-aminosalicylic acid. CrystEngComm 2011, 13, 4358-4364.

8. Sarma, B.; Saikia, B. Hydrogen bond synthon competition in the stabilization of theophylline cocrystals. CrystEngComm 2014, 16, 4753-4765.

9. Regulatory Classification of Pharmaceutical Co-Crystals. Available online: http://www.fda.gov/ downloads/drugs/guidancecomplianceregulatoryinformation/guidances/ucm281764.pdf (accessed on 11 September 2015).

10. Eddleston, M.D.; Sivachelvam, S.; Jones, W. Screening for polymorphs of cocrystals: A case study. Cryst. Eng. Comm. 2013, 15, 175-181.

11. Braatz, R.D.; Fujiwara, M.; Wubben, T.J.; Rusli, E. Crystallization: Particle size control. In Encyclopedia of Pharmaceutical Technology, 3rd ed.; Swarbrick, J., Ed.; Informa Healthcare: New York, NY, USA, 2007; Volume 2, pp. 858-871.

12. Imamura, M.; Nakanishi, K.; Shiraki, R.; Onda, K.; Sasuga, D.; Yuda, M. Cocrystal of C-Glycoside Derivative and L-Proline. U.S. Patent 20090143316 A1, 4 June 2009.

13. Trask, A.V.; Motherwell, W.D.S.; Jones, W. Pharmaceutical cocrystallization: Engineering a remedy for caffeine hydration. Cryst. Growth Des. 2005, 5, 1013-1021.

14. Karki, S.; Friščić, T.; Fábián, L.; Laity, P.R.; Day, G.M.; Jones, W. Improving mechanical properties of crystalline solids by cocrystal formation: New compressible forms of paracetamol. Adv. Mater. 2009, 21, 3905-3909.

15. Shevchenko, A.; Bimbo, L.M.; Miroshnyk, I.; Haarala, J.; Jelínková, K.; Syrjänen, K.; van Veen, B.; Kiesvaara, J.; Santos, H.A.; Yliruusi, J. A new cocrystal and salts of itraconazole: Comparison of solid-state properties, stability and dissolution behavior. Int. J. Pharm. 2012, 436, 403-409.

16. Jung, S.; Lee, J.; Kim, I.W. Structures and physical properties of the cocrystals of adefovir dipivoxil with dicarboxylic acids. J. Cryst. Growth 2013, 373, 59-63.

17. Zügner, S.; Marquardt, K.; Zimmermann, I. Influence of nanomechanical crystal properties on the comminution process of particulate solids in spiral jet mills. Eur. J. Pharm. Biopharm. 2006, 62, 194-201.

18. Meier, M.; John, E.; Wieckhusen, D.; Wirth, W.; Peukert, W. Influence of mechanical properties on impact fracture: Prediction of the milling behaviour of pharmaceutical powders by nanoindentation. Powder Technol. 2009, 188, 301-313.

19. Chattoraj, S.; Shi, L.; Changquan, C.S. Understanding the relationship between crystal structure, plasticity and compaction behaviour of theophylline, methyl gallate, and their 1:1 co-crystal. Cryst. Eng. Comm. 2010, 12, 2466-2472.

20. Arimilli, M.N.; Kelly, D.E.; Lee, T.T.K.; Manes, L.V.; Munger, J.D., Jr.; Prisbe, E.J.; Schultze, L.M. Nucleotide Analog Compositions. U.S. Patent 20020045599 A1, 18 April 2002.

21. Jung, S.; Kim, I.W. Effects of polymers on the cocrystallization of adefovir dipivoxil and suberic acid. Polym. Korea 2013, 37, 663-668. 
22. Gao, Y.; Zu, H.; Zhang, J. Enhanced dissolution and stability of adefovir dipivoxil by cocrystal formation. J. Pharm. Pharmacol. 2011, 63, 483-490.

23. Jung, S.; Ha, J.-M.; Kim, I.W. Phase transformation of adefovir dipivoxil/succinic acid cocrystals regulated by polymeric additives. Polymers 2014, 6, 1-11.

24. Bailey Walsh, R.D.; Bradner, M.W.; Fleischman, S.; Morales, L.A.; Moulton, B.; Rodríguez-Hornedo, N.; Zaworotko, M.J. Crystal engineering of the composition of pharmaceutical phases. Chem. Commun. 2003, 186-187.

25. Noyes, A.A.; Whitney, W.R. The rate of solution of solid substances in their own solutions. J. Am. Chem. Soc. 1897, 11, 930-934.

26. Dokoumetzidis, A.; Macheras, P. A century of dissolution research: From Noyes and Whitney to the biopharmaceutics classification system. Int. J. Pharm. 2006, 321, 1-11.

(C) 2015 by the authors; licensee MDPI, Basel, Switzerland. This article is an open access article distributed under the terms and conditions of the Creative Commons Attribution license (http://creativecommons.org/licenses/by/4.0/). 